\title{
NONCOMMUTING QUASIGROUP CONGRUENCES
}

\author{
H. A. THURSTON
}

1. The purpose of this paper is to exhibit a quasigroup with two noncommuting congruences on it. The quasigroup is in fact the free equationally-defined commutative quasigroup ${ }^{1}$ generated by four elements, and I shall use the construction devised by G. E. Bates and F. Kiokemeister, Bull. Amer. Math. Soc. vol. 54 (1948) p. 1180.

2. Definition. A set $S$ of elements such that to each of certain pairs $a, b$ of elements there corresponds a uniquely-defined product $a b$ in $S$ and such that if $a b$ is defined then so is $b a$ and is equal to it is a partial commutative groupoid. The identity $x y=y x$ will be implicitly assumed; e.g. if I define $p q$, then $q p$ is to be defined as the same element, even if this is not explicitly mentioned.

3. Definition. $T$ is the extension of a partial commutative groupoid $S$ if $T$ consists of the elements of $S$, together with an element $a \times b$ for each pair $a, b$ for which $a b$ is not defined in $S$ and an element $a / b$ for each ordered pair $a, b$ for which $b x=a$ is not solvable in $S$, $a \times b$ being equal to $b \times a$, but all other elements being distinct. Multiplication is defined in $T$ as follows: if $a b$ is defined in $S$, then it is defined in $T$ to be the same element; if $a b$ is not defined in $S$, then $a b$ is defined in $T$ to be $a \times b$; and for each $a / b$ defined as above $(a / b) b=a$. All other products in $T$ are undefined.

4. Let $J_{0}$ be a commutative partial groupoid in which no products are defined. For each $i$, let $J_{i+1}$ be the extension of $J_{i}$, and let $M$ be $\bigcup_{i>0} J_{i}$. Then $M$ is a commutative quasigroup (op. cit. Corollary 2).

Definition. The rank, $R_{x}$, of an element $x$ of $M$ is the suffix of the first $J_{i}$ to which $x$ belongs.

(We could complete the definition of division as an operation on $M$ by putting $(x y) / y$ equal to $x$. If we do this we see that the algebra we have defined is in fact the free equationally-defined quasigroup generated by the elements of $J_{0}$.)

5. Let $q_{i}$ be a congruence on $J_{i}$; that is, an equivalence on $J_{i}$ such that if $a \mathfrak{q} a^{\prime}$ and $b \mathfrak{q} b^{\prime}$ and $a b \in J_{i}$ and $a^{\prime} b^{\prime} \in J_{i}$, then $a b \mathfrak{q}_{i} a^{\prime} b^{\prime}$. We define $\mathfrak{q}_{i+1}$ as follows: $x \mathfrak{q}_{i+1} y$ and $y \mathfrak{q}_{i+1} x$ if and only if

Received by the editors March 26, 1951 and, in revised form, September 19, 1951.

1 The congruences are quasigroup congruences, not equationally-defined-quasigroup congruences (it is known that any two of the latter commute). (See the last sentence but one of $\$ 5$.) 
(i) $x q_{i} y$,

(ii) $x=y\left(R_{x}=i+1\right)$,

(iii) $x=a \times b$ and $y=a^{\prime} \times b^{\prime}$, where $a q_{i} a^{\prime}$ and $b q_{i} b^{\prime}$,

(iv) $x=a \times b$ and $y q_{i} a^{\prime} b^{\prime}$ where $a q_{i} a^{\prime}$ and $b q_{i} b^{\prime}$, or

(v) $x \mathfrak{q}_{i+1} z$ and $y \mathfrak{q}_{i+1} z^{\prime}$ via (iv), and $z \mathfrak{q}_{i} z^{\prime}$.

(i) ensures that $\mathfrak{q}_{i+1} \supseteq \mathfrak{q}_{i}$; (ii) ensures that $\mathfrak{q}_{i+1}$ is on $J_{i+1}$; (iv) gives the conditions under which an element of rank $i+1$ is equivalent to one of lower rank; and (v) says that two elements of rank $i+1$ which are equivalent to two equivalent elements in $J_{i}$ are equivalent to one another, and so ensures that $q_{i+1}$ is transitive. In fact, $q_{i+1}$ is an equivalence on $J_{i+1}$, and if $a$ and $b$ are in $J_{i}$, then $a q_{i+1} b$ if and only if $a q_{i} b$.

$\mathfrak{q}_{i+1}$ is a congruence. For suppose that $a \mathfrak{q}_{i+1} a^{\prime}, b \mathfrak{q}_{i+1} b^{\prime}, a b \in J_{i+1}$, and $a^{\prime} b^{\prime} \in J_{i+1}$. If $a, a^{\prime}, b, b^{\prime} \in J_{i}$, then $a b \mathfrak{q}_{i+1} a^{\prime} b^{\prime}$; this follows from the fact that $\mathfrak{q}_{i}$ is a congruence if $a b$ and $a^{\prime} b^{\prime}$ are in $J_{i}$, from (iii) if neither is in $J_{i}$, and from (iv) if just one is. Now suppose that $a$ is not in $J_{i}$. Since $a b$ is in $J_{i+1}, a$ must be of the form $c / b$, where $c \in J_{i}$. $c / b$ is equivalent only to itself, for, of (i) to (v), only (ii) applies to elements of this form. Therefore $a^{\prime}=c / b$. But $a^{\prime} b^{\prime}$ is in $J_{i+1}$. Therefore $b^{\prime}=b$. Then $a b=c=a^{\prime} b^{\prime}$. Similarly we see that $a b q_{i+1} a^{\prime} b^{\prime}$ if any other of the elements $a, a^{\prime}, b, b^{\prime}$ is not in $J_{i}$.

It follows that if $q_{0}$ is a congruence on $J_{0}$ and $q_{i}$ is defined for each $i>0$ as above and $q=U_{i>0} q_{i}$, then $q$ is a congruence on $M$. It is in fact the least congruence on $M$ for which $a \mathfrak{q} b$ whenever $a q_{0} b$. (It is a congruence for multiplication only, not for division, unless $q_{0}$ is equality.) Clearly $\mathfrak{q} \cap\left(J_{i} \times J_{i}\right)=\mathfrak{q}_{i}$.

6. An example will illustrate this definition. Let $J_{0}$ be $\{\alpha, \beta, \gamma, \delta\}$ and $q_{0}$ be $\alpha \beta|\gamma| \delta$. (This notation means that the $q_{0}$-classes are $\{\alpha, \beta\},\{\gamma\}$, and $\{\delta\}$.) The columns of the table show the $q$-classes; the rows show the rank of the entry.

\begin{tabular}{|c|c|c|c|}
\hline 0 & $\alpha, \beta$ & $\gamma$ & $\delta$ \\
\hline 1 & & & 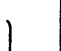 \\
\hline 2 & $(\alpha / \beta) \alpha,(\beta / \alpha) \beta$, etc. & $(\gamma / \alpha) \beta,(\gamma / \beta) \alpha$ & $a_{\gamma}$ \\
\hline 3 & $(\alpha / \alpha \alpha)(\alpha \beta)$ etc. & $(\gamma / \alpha \alpha)(\alpha \beta)$ etc. & . \\
\hline
\end{tabular}




\begin{tabular}{c|c|c|c|c|c|c|c}
\hline 1 & $\alpha \alpha, \alpha \beta, \beta \beta$ & $\alpha \gamma, \beta \gamma$ & $\ldots$ & $\alpha / \alpha$ & $\ldots$ & & \\
\hline 2 & & & & & & $\alpha \alpha \cdot \alpha, \alpha \alpha \cdot \beta, \alpha \beta \cdot \alpha$, etc. & $\ldots$ \\
\hline 3 & $\frac{\alpha \alpha}{\beta} \alpha$ etc. & $\frac{\alpha \gamma}{\beta} \alpha$ etc. & $\ldots$ & $\frac{\alpha / \alpha}{\alpha} \beta$ etc. & $\ldots$ & & \\
\hline & $\vdots$ & $\vdots$ & $\vdots$ & $\vdots$ & $\vdots$
\end{tabular}

The process of constructing the table is roughly this: the first row is given. In the second row, no element can go into one of the existing classes, for an element can be equivalent to an element of a previous $J_{i}$ only via (iv): this requires that the previous element factorizes; but no element of $J_{0}$ factorizes in $J_{0}$. The elements $\alpha \alpha, \alpha \beta$, and $\beta \beta$ are gathered into one class by (iii), so are $\alpha \gamma$ and $\beta \gamma$, etc. When we come to $J_{2}$, since $\alpha=(\alpha / \alpha) \alpha=(\alpha / \beta) \beta$ we get $(\alpha / \beta) \alpha$ and $(\alpha / \alpha) \beta$ in the $q$-class of $\alpha$, and so on.

7. Theorem. Let $\mathfrak{q}$ etc. be as above, and let $\mathfrak{r}$ be defined similarly by putting $\mathfrak{r}_{0}=\alpha|\beta| \gamma \delta$. If $a \mathfrak{q} c \mathfrak{r} b$ there is a $d$ of rank less than or equal to $\max \left\{R_{a}, R_{b}\right\}$ such that $a \mathfrak{q} d \mathfrak{r} b$.

Proof. Let $\Phi_{n}$ be the statement "If $a, b$, and $c$ are in $J_{n}$ and if $a \mathfrak{q} c \mathfrak{r} b$, then $a \mathfrak{q} d \mathfrak{r} b$ where $\mathcal{R}_{d} \leqq \max \left\{\mathcal{R}_{a}, \mathcal{R}_{b}\right\} . " \Phi_{n}$ may be proved by induction. $\Phi_{0}$ is clearly true, and so is $P_{1}$. Let $n>1$ and suppose $\mathbb{P}_{m}$ true whenever $m<n$. Of all the elements $x$ for which $a \mathfrak{q} x \mathfrak{r} b$, let $c$ be one of least rank. If $\max \left\{R_{a}, R_{b}, R_{c}\right\}<n$, then $\mathcal{P}_{n}$ is true by the induction hypothesis. If $\max \left\{R_{a}, R_{b}\right\}=n$, then $P_{n}$ is clearly true. We are left with the case $R_{c}=n, R_{a}<n, R_{b}<n$.

Then $c$ is equivalent to an element $a$ of lower rank. Therefore, by $\S 5$ (iii), $c=d e$ and $a=d^{\prime} e^{\prime}$, where $d \mathfrak{q}_{n-1} d^{\prime}$ and $e \mathfrak{q}_{n-1} e^{\prime}$. Also $\max \left\{R_{d}, R_{e}\right\}=n-1$, otherwise we would not have $R_{c}=n$. Similarly $b=d^{\prime \prime} e^{\prime \prime}$, where $d \mathfrak{r}_{n-1} d^{\prime \prime}$ and $e \mathfrak{r}_{n-1} e^{\prime \prime}$.

Now we apply $\Phi_{n-1}$ to $d^{\prime}, d$, and $d^{\prime \prime}$. There exists then a $d^{\prime \prime \prime}$ in $J_{n-1}$ such that

$$
d^{\prime} \mathfrak{q} d^{\prime \prime \prime} \mathfrak{r} d^{\prime \prime} \text { and } R_{d^{\prime \prime}} \leqq \max \left\{\mathcal{R}_{d^{\prime}}, R_{d^{\prime \prime}}\right\} .
$$

Similarly,

$$
e^{\prime} \mathfrak{q} e^{\prime \prime \prime} \mathfrak{r} e^{\prime \prime} \text { and } R_{e^{\prime \prime \prime}} \leqq \max \left\{R_{e^{\prime}}, R_{e^{\prime \prime}}\right\} .
$$

Now $a \mathfrak{q} d^{\prime \prime \prime} e^{\prime \prime \prime} \mathfrak{r} b$. Since $c$ is an element of least rank for which $a \mathfrak{q} c$ $\mathfrak{r} b$, we have $R_{d^{\prime \prime \prime} e^{\prime \prime \prime}} \geqq n$, whence clearly $R_{d^{\prime \prime \prime} e^{\prime \prime \prime}}=n$. Then $\max$ $\left\{R_{d^{\prime \prime \prime}}, R_{e^{\prime \prime}}\right\}=n-1$. Suppose it is $d^{\prime \prime \prime}$ which is of rank $n-1$. Then 


$$
\begin{aligned}
n-1=R_{d^{\prime \prime \prime}} & \leqq \max \left\{R_{d^{\prime}}, R_{d^{\prime \prime}}\right\} \quad \text { (by (1)) } \\
& \leqq n-1 \quad \text { (because } d^{\prime} \text { and } d^{\prime \prime} \text { are in } J_{n-1} \text { ). }
\end{aligned}
$$

Therefore one of $d^{\prime}, d^{\prime \prime}$ is of rank $n-1$; suppose it is $d^{\prime}$. We have now that $d^{\prime} e^{\prime} \in J_{n-1}$ and $d^{\prime}$ is of rank $n-1$. Then we must have $d^{\prime}=f / e^{\prime}$. We saw in $\$ 4$ that an element of this form of rank $n-1$ is not equivalent to any other element of $J_{n-1}$. Therefore $d^{\prime}=d^{\prime \prime}=d^{\prime \prime \prime}=f / e^{\prime}$, where $R_{f}<n-1$. Now $\left(f / e^{\prime}\right) e^{\prime \prime}=d^{\prime \prime} e^{\prime \prime} \in J_{n-1}$. Therefore $e^{\prime}=e^{\prime \prime}$. Therefore $d^{\prime} e^{\prime}=d^{\prime \prime} e^{\prime \prime}=f$. Therefore $a \mathfrak{q} f \mathfrak{r} b$ and $R_{r}<n-1$. This contradicts the definition of $c$.

8. In the theorem of $\S 7$, put $a=\delta$ and $b=(\gamma / \alpha) \beta$. Then $\max \left\{R_{a}, R_{b}\right\}=2$. Therefore if there is a $c$ such that $a \mathfrak{q} c \mathfrak{r} b$, there will be one whose rank is at most 2. Clearly there is no such $c$.

On the other hand, $a=\delta \mathfrak{r} \gamma \mathfrak{q}(\gamma / \alpha) \beta=b$. Therefore $\mathfrak{q}$ and $\mathfrak{r}$ do not commute.

The theorem of $\$ 7$ is the application to this problem of a theorem (as yet unpublished) of J. C. Shepherdson.

The reader will notice that $\mathfrak{q}$ and $\mathfrak{r}$ have an infinite number of infinite congruence classes. This is important. I have just received a proof from S. Abhyankar, Harvard University, of the fact that if $q$ and $\mathfrak{r}$ both fail to have this doubly infinite character, then they commute.

UNIVERSITY OF BRISTOL 